\title{
Numerical errors generated by WENO-based interface-capturing schemes in multifluid computations
}

\author{
Eric Johnsen * \\ University of Michigan, Ann Arbor, MI 48109, USA \\ Johan Larsson ${ }^{\dagger}$ \\ Stanford University, Stanford, CA 93405, USA
}

\begin{abstract}
When using high-order accurate shock-capturing methods, e.g., weighted essentially non-oscillatory schemes (WENO), numerical errors are often generated in simulations of isolated contact discontinuities and material interfaces when trying to capture these features on a grid. Spurious pressure oscillations are generated at interfaces and contacts propagate through the flow field. In addition, temperature errors may be generated. The present work constitutes an attempt to provide a unifying theory describing these errors. First, contact discontinuities in a single-component fluid are discussed, followed by material interfaces and contact discontinuities separating different fluids. Errors that are generated in simulations of these features are analyzed and means to prevent them are introduced and discussed.
\end{abstract}

\section{Nomenclature}

$c_{v}=$ specific heat at constant volume

$c_{p}=$ specific heat at constant pressure

$e=$ internal energy per unit mass

$E=$ total energy

$M=$ molecular mass

$p=$ pressure

$R=$ gas constant

$s \quad=$ wave speed

$T=$ temperature

$u=$ velocity

$z=$ mass fraction

$\gamma=$ ratio of specific heats

$\rho=$ density

\section{Introduction}

In a wide range of compressible flows, density discontinuities across which the velocity and pressure are constant occur. In one dimension, they are of three types: single-component contact discontinuities (discontinuous temperature, constant composition), material interfaces (discontinuous composition, constant temperature) and contact discontinuities separating different fluids (discontinuous temperature and composition). Hence, the density is discontinuous across each these features, but the velocity and pressure are constant. In many single-component compressible flows, shock waves interact with each other, thus pro-

*Assistant Professor, Mechanical Engineering Department, AIAA Member.

${ }^{\dagger}$ Research Associate, Center for Turbulence Research, AIAA Member. 
ducing contact discontinuities. In high-speed combustion applications, fluids of different composition and sometimes temperature are present, and shock waves interact with them.

The numerical treatment of such density discontinuities is not trivial. Shock-capturing schemes must usually be modified to provide accurate solutions to problems with contact discontinuities or material interfaces. When trying to capture these features on a grid, high-order accurate shock-capturing schemes usually exhibit velocity and pressure oscillations across isolated single-component contact discontinuities, ${ }^{7}$ material interfaces ${ }^{1,6}$ and contact discontinuities separating different fluids. ${ }^{8}$ Furthermore, temperature errors may be generated, ${ }^{8}$ though this matter is not of concern for Euler calculations.

The goal of the present work is to provide a synthesis of numerical errors generated in WENO simulations of all density discontinuities across which the velocity and pressure are constant (i.e., contacts and interfaces), given prior findings on this topic. ${ }^{1,6-8,11}$ In particular, pressure and temperature errors are discussed. The focus is on isolated discontinuities, with no interaction with other flow features. First, the numerical formulation is described, including the equations of motion and the numerical scheme. Then, a one-dimensional analysis of the errors and means to prevent them are presented.

\section{Numerical formulation}

\section{II.A. Equations of motion}

The equations of conservation of mass, momentum and energy for a system of two gases in which no diffusion takes place are given by:

$$
\begin{aligned}
& \frac{\partial \rho}{\partial t}+\frac{\partial}{\partial x_{j}}\left(\rho u_{j}\right)=0, \\
& \frac{\partial\left(\rho u_{i}\right)}{\partial t}+\frac{\partial}{\partial x_{j}}\left(\rho u_{i} u_{j}+p \delta_{i j}\right)=0, \\
& \frac{\partial E}{\partial t}+\frac{\partial}{\partial x_{j}}\left[u_{j}(E+p)\right]=0, \\
& \frac{\partial(\rho z)}{\partial t}+\frac{\partial}{\partial x_{j}}\left(\rho z u_{j}\right)=0,
\end{aligned}
$$

where $u$ is the velocity vector, $p$ is the pressure, $E=\rho\left(e+u^{2} / 2\right)$ is the total energy per unit volume, $h=(e+p) / \rho$ is the enthalpy and $\delta_{i j}$ is the identity tensor. The transport equation is included when the fluid composition varies; it is written here in conservative form, but can also be expressed in advection form. The system is closed using the ideal gas law,

$$
\frac{p}{\gamma-1}=\rho e
$$

where $\gamma=c_{p} / c_{v}$ is the ratio of specific heats, $e=c_{v} T$ is the internal energy and $T$ the temperature.

For varying fluid composition, non-reacting gases are considered. To simplify the analysis, two gases, denoted by superscripts $\alpha$ and $\beta$, are considered. The mass fraction of the first gas is $z=z^{\alpha}$. Since two gases

are considered, a single mass fraction is required. The mixture molecular mass follows from the definition of the mass fraction:

$$
\frac{1}{M}=\frac{z}{M^{\alpha}}+\frac{1-z}{M^{\beta}}
$$

The ideal gas law can also be written

$$
p=\rho R T,
$$

where $R$ is the gas constant given by

$$
R=\frac{R_{u}}{M}=R^{\alpha} z+R^{\beta}(1-z) .
$$

Here, $R_{u}$ is the universal gas constant. It follows from the definition of $R$ that the specific heats are given by

$$
c_{v}=c_{v}^{\alpha} z+c_{v}^{\beta}(1-z), \quad c_{p}=c_{p}^{\alpha} z+c_{p}^{\beta}(1-z) .
$$


Finally, the following relation will be useful in this work:

$$
\frac{1}{\gamma-1}=\frac{z}{\gamma^{\alpha}-1} \frac{M}{M^{\alpha}}+\frac{1-z}{\gamma^{\beta}-1} \frac{M}{M^{\beta}} .
$$

\section{II.B. Numerical method}

In the present work, a high-order accurate discretization scheme is used for the convective terms. For a conservation law of the type

$$
q_{t}+[f(q)]_{x}=0,
$$

the finite volume discretization yields the following semi-discrete form:

$$
\frac{d}{d t} \bar{q}_{i}=-\frac{\hat{f}_{i+1 / 2}-\hat{f}_{i-1 / 2}}{\Delta x}
$$

where the overbar refers to the cell-average value and $\hat{f}_{i+1 / 2}$ is the numerical flux. A typical algorithm for finite volume shock-capturing schemes is as follows: given the cell average values, $\bar{q}_{i}$, reconstruct the pointwise left and right values at the cell edges, $q_{i+1 / 2}^{L}$ and $q_{i+1 / 2}^{R}$; then, compute the numerical flux using an approximate Riemann solver, $h$, with $\hat{f}_{i+1 / 2}=h\left(q_{i+1 / 2}^{L}, q_{i+1 / 2}^{R}\right)$. The solution is then marched forward in time.

For the time marching, a third-order accurate total variation diminishing (TVD) Runge-Kutta scheme ${ }^{4}$ is used. For the spatial discretization a high-order accurate finite volume WENO reconstruction ${ }^{9}$ is employed with Riemann solvers of the HLL family, ${ }^{5}$ including Lax-Friedrichs solvers. The numerical flux is given by:

$$
f_{i+1 / 2}=\frac{s_{i+1 / 2}^{+} f\left(q_{i+1 / 2}^{R}\right)-s_{i+1 / 2}^{-} f\left(q_{i+1 / 2}^{L}\right)}{s_{i+1 / 2}^{+}-s_{i+1 / 2}^{-}}-\frac{s_{i+1 / 2}^{+} s_{i+1 / 2}^{-}}{s_{i+1 / 2}^{+}-s_{i+1 / 2}^{-}}\left(q_{i+1 / 2}^{R}-q_{i+1 / 2}^{L}\right),
$$

where $s_{i+1 / 2}^{+}=\max \left(0, s_{i+1 / 2}^{R}\right)$ and $s_{i+1 / 2}^{-}=\min \left(0, s_{i+1 / 2}^{L}\right)$. The wave speeds $s^{R}$ and $s^{L}$ are determined based on Einfeldt et al. (1991). ${ }^{3}$ For Lax-Friedrichs, $s_{i+1 / 2}^{+}=-s_{i+1 / 2}^{-}=\alpha=\max _{i, l}\left|\lambda_{i}^{(l)}\right|$. For the transport equation in advection form, such schemes must be modified. ${ }^{10}$

For simplicity and without loss of generality, the third-order accurate WENO reconstruction of the celledge value, $v_{i+1 / 2}^{L}$, of a polynomial, $v(x)$, from the cell-average values, $\bar{v}_{j}$, where $j=[i-1, i, i+1]$, is considered. The reconstructed value is given by:

$$
v_{i+1 / 2}^{L}=\frac{\alpha_{0}}{\alpha_{0}+\alpha_{1}} p_{0}+\frac{\alpha_{1}}{\alpha_{0}+\alpha_{1}} p_{1}=\frac{1}{1+\alpha_{1} / \alpha_{0}} p_{0}+\frac{1}{1+\alpha_{0} / \alpha_{1}} p_{1},
$$

where the linear interpolating polynomials are given by

$$
p_{0}=\frac{3}{2} \bar{v}_{i}-\frac{1}{2} \bar{v}_{i+1}, \quad p_{1}=\frac{1}{2} \bar{v}_{i-1}+\frac{1}{2} \bar{v}_{i} .
$$

The nonlinear weights are given by

$$
\alpha_{0}=\frac{d_{0}}{\left(\beta_{0}+\epsilon\right)^{2}}, \quad \alpha_{1}=\frac{d_{1}}{\left(\beta_{1}+\epsilon\right)^{2}},
$$

with ideal weights $d_{0}=1 / 3$ and $d_{1}=2 / 3$. The smoothness indicators are given by

$$
\beta_{0}=\left(v_{i+1}-v_{i}\right)^{2}, \quad \beta_{1}=\left(v_{i}-v_{i-1}\right)^{2} .
$$

\section{Analysis of the numerical errors in flows with density discontinuities}

Historically, shock-capturing schemes have been designed to provide stable solutions to flows with shocks in a single fluid. In the present section, conditions that must be satisfied to prevent numerical errors at interfaces and contact discontinuities are derived. Contacts and interfaces are characterized by a discontinuous density, but with constant velocity and pressure. Three cases are possible: a contact discontinuity 
in a single-component fluid, where the temperature is discontinuous, but the mass fraction is constant; a material interface separating different fluids, where the mass fraction is discontinuous, but the temperature is constant; and a contact discontinuity separating different fluids, where both the mass fraction and the temperature are discontinuous. In flows in which shock waves interact with interfaces, e.g., Richtmyer-Meshkov instability, ${ }^{2}$ all three phenomena may be present.

To illustrate the numerical errors and motivate the proposed scheme, a one-dimensional discontinuity in helium or between helium and nitrogen moves at a constant velocity with uniform pressure. The variables are non-dimensionalized by the density, $\rho_{H e}$, and sound speed, $c_{H e}$, of helium, and the domain length, $L$. The initial conditions are as follows:

$$
\rho / \rho_{H e}=\frac{p_{a} / R T}{p_{a} / R_{H e} T_{a}}, \quad u / c_{H e}=1, \quad p / \rho_{H e} c_{H e}^{2}=1 / \gamma_{H e},
$$

where $R$ is given by equation (5). The mass fraction and temperature are set as follows:

- Contact discontinuity in a single fluid: $z=1$ and

$$
T / T_{o}= \begin{cases}2, & \text { if }-0.5 \leq x \leq 0.5 \\ 1, & \text { otherwise }\end{cases}
$$

- Material interface separating different fluids (of different $\gamma$ ): $T / T_{o}=1$ and

$$
z_{H e}= \begin{cases}0, & \text { if }-0.5 \leq x \leq 0.5 \\ 1, & \text { otherwise. }\end{cases}
$$

- Contact discontinuity separating different fluids (of different $\gamma$ ):

$$
T / T_{o}=\left\{\begin{array}{ll}
2, & \text { if }-0.5 \leq x \leq 0.5, \\
1, & \text { otherwise }
\end{array} \quad z_{H e}= \begin{cases}0, & \text { if }-0.5 \leq x \leq 0.5 \\
1, & \text { otherwise }\end{cases}\right.
$$

The initial discontinuity consists of a one-point jump. Both $u$ and $p$ are expected to remain constant for all time, as there is no diffusion. Since the Euler equations are considered, the temperature only enters the problem through the initial conditions and is passively transported.

The Euler equations are analyzed in order to determine the appropriate form of the transport equation. ${ }^{1}$ If a discontinuity is located in cell $i$, the mass, momentum and energy conservation equations can be marched forward by one time step or substep:

$$
\begin{aligned}
\rho_{i}^{n+1} & =\rho_{i}^{n}-\frac{\Delta t}{\Delta x} D_{i}(\rho u), \\
(\rho u)_{i}^{n+1} & =(\rho u)_{i}^{n}-\frac{\Delta t}{\Delta x}\left[D_{i}\left(\rho u^{2}\right)+D_{i}(p)\right], \\
\left(\rho e+\rho \frac{u^{2}}{2}\right)_{i}^{n+1} & =\left(\rho e+\rho \frac{u^{2}}{2}\right)_{i}^{n}-\frac{\Delta t}{\Delta x}\left[D_{i}(\rho u e)+D_{i}\left(\rho \frac{u^{3}}{2}\right)+D_{i}(u p)\right] .
\end{aligned}
$$

For the time being, the difference operator, $D_{i}(\cdot)$, is assumed to have the following properties: $D_{i}(a b+$ $c)=a D_{i}(b)+D_{i}(c)$ for $a$ constant, $b$ and $c$ not constant; thus, the chain rule does not apply discretely: $D_{i}(b c) \neq b_{i} D_{i}(c)+c_{i} D_{i}(b)$. The operator $D_{i}$ thus includes the reconstruction step and the upwinding. The standard WENO procedure in which the conservative variables are reconstructed does not satisfy this property.

For constant $u$ and $p$ and $D_{i}(p), D_{i}(u)=0$, equations (19) reduce to

$$
\begin{aligned}
\rho_{i}^{n+1} & =\rho_{i}^{n}-\frac{\Delta t}{\Delta x} u D_{i}(\rho), \\
(\rho u)_{i}^{n+1} & =(\rho u)_{i}^{n}-\frac{\Delta t}{\Delta x} u^{2} D_{i}(\rho), \\
\left(\rho e+\rho \frac{u^{2}}{2}\right)_{i}^{n+1} & =\left(\rho e+\rho \frac{u^{2}}{2}\right)_{i}^{n}-\frac{\Delta t}{\Delta x} u\left[D_{i}(\rho e)+\frac{u^{2}}{2} D_{i}(\rho)\right] .
\end{aligned}
$$


The mass and momentum equations can be combined to yield

$$
u_{i}^{n+1}=u_{i}^{n}=u,
$$

and the energy equation can be written

$$
(\rho e)_{i}^{n+1}=(\rho e)_{i}^{n}-\frac{\Delta t}{\Delta x} u D_{i}(\rho e) .
$$

It is emphasized that equation (22) holds only if $D_{i}(p)=0$ and $D_{i}(u)=0$. This condition is not trivial and is discussed in section III.A.

Assuming that $D_{i}$ can be modified to satisfy the above properties, the goal of the rest of this section is to determine how the transport equation must be solved in order to prevent numerical errors in pressure, temperature and mass fraction. First, a contact discontinuity in a single fluid is considered, to illustrate that $D_{i}(a b) \neq a D_{i}(b)$ for $a$ constant, but $b$ not constant in WENO methods. Then, two different viewpoints are followed to simulate material interfaces without generating errors when solving Equation (22): using either Equation (2) relating the pressure to the internal energy (pressure viewpoint ${ }^{1,11}$ ), or using Equation (4) to relate the temperature to the internal energy (temperature viewpoint). Finally, a scheme capable of dealing with contact discontinuities between different fluids, in addition to contact discontinuities in a single fluid and material interfaces, is proposed.

\section{III.A. Advection of a contact discontinuity in a single fluid: WENO reconstruction of the average primitive variables}

Numerical errors associated with the advection of a contact in a single-component fluid have been examined by Johnsen (2011a). ${ }^{7}$ In this section, the key findings are summarized and expanded to provide context for errors that occur when the fluid composition is discontinuous.

For the advection of an isolated contact discontinuity, the mass, momentum and energy equations are all expected to reduce to the same equation: the advection equation for density, since velocity and pressure are constant, i.e.,

$$
\rho_{i}^{n+1}=\rho_{i}^{n}-\frac{\Delta t}{\Delta x} u D_{i}(\rho) .
$$

However, when performing the WENO reconstruction, it is easy to show that the left reconstructed density is inconsistent in the three equations ${ }^{7}$

$$
\rho_{i+1 / 2}^{L} \neq \frac{\rho u_{i+1 / 2}^{L}}{u} \neq \frac{\rho u_{i+1 / 2}^{L, 2} / 2}{u^{2} / 2} .
$$

In fact, the errors are of order $O(\epsilon)$. As a result, the reconstructed velocity and pressure are no longer constant in the flow field.

It is easy to show that the velocity and pressure at the following time step are given by: ${ }^{7}$

$$
\begin{gathered}
u_{i}^{n+1}=\frac{\rho u_{i}^{n}-\frac{\Delta t}{\Delta x} D_{i}^{\rho u}(\rho u u)}{\rho_{i}^{n}-\frac{\Delta t}{\Delta x} D_{i}^{\rho}(\rho u)}-\frac{\frac{\Delta t}{\Delta x} D_{i}(p)}{\rho_{i}^{n}-\frac{\Delta t}{\Delta x} D_{i}^{\rho}(\rho u)}, \\
p_{i}^{n+1}=(\gamma-1)_{i}^{n+1}\left[\frac{p_{i}^{n}}{(\gamma-1)_{i}^{n}}-\frac{\Delta t}{\Delta x} D_{i}^{E}\left(\frac{p}{\gamma-1} u\right)-\frac{\Delta t}{\Delta x} D_{i}(u p)+\frac{\rho u_{i}^{2}}{2}-\frac{\Delta t}{\Delta x} D_{i}^{E}\left(\rho \frac{u^{2}}{2} u\right)\right. \\
\left.-\frac{1}{2} \frac{\left\{\rho u_{i}^{n}-\frac{\Delta t}{\Delta x}\left[D_{i}^{\rho u}(\rho u u)+D_{i}(p)\right]\right\}^{2}}{\rho_{i}^{n}-\frac{\Delta t}{\Delta x} D_{i}^{\rho}(\rho u)}\right] .
\end{gathered}
$$

It is clear that the last term in Equation (25) and the last five terms in Equation (26) do not cancel out, such that the velocity and pressure at the next time step are no longer constant. Thus, errors are generated at the first time step and continue to grow with time, up to approximately $0.1 \%{ }^{7}$ By choosing an appropriate value of $\epsilon$ that is different for each variable, the errors in the velocity and pressure can be delayed. For example, in the case of $u=1, \rho u_{i+1 / 2}^{L}=\rho_{i+1 / 2}^{L} u$ and $\epsilon$ in the energy can be divided by 4 . However, this will only delay the error growth, not suppress it. 
In order to prevent this error, a single condition must be satisfied: the reconstructed density must be consistent in all three equations. Two important consequences follow: (i) the different terms including density in Equations (25) and (26) cancel out, and (ii) the the reconstructed velocity and pressure remain constant, so that the relevant terms cancel. By carefully choosing the variables to be reconstructed, this condition can be met. Namely, a reconstruction of the primitive variables or the characteristic variables yields this result, though for different reasons. In the primitive variable reconstruction, the density, velocity and pressure are reconstructed, thus providing a unique and consistent value for the density and constant velocity and pressure. In the characteristic variable reconstruction, the transformation to characteristic space using the eigenvector matrix

$$
R=\left(\begin{array}{ccc}
1 & 1 & 1 \\
u-a & u & u+a \\
h-u a & k & h+u a
\end{array}\right), \quad h=\frac{a^{2}}{\gamma-1}+k, \quad k=\frac{u^{2}}{2}
$$

leads to the reconstruction of the following variables:

$$
R^{-1} q=\left(\frac{\gamma-1}{2 a^{2}} \frac{p}{\gamma-1}, \quad \rho-\frac{\gamma-1}{a^{2}} \frac{p}{\gamma-1}, \quad \frac{\gamma-1}{2 a^{2}} \frac{p}{\gamma-1}\right)^{T} .
$$

To understand the mechanism that allows the characteristic reconstruction to work, consider the first component of the reconstructed variable:

$$
R\left[R^{-1} q\right]_{i+1 / 2}^{(1)}=\frac{\gamma-1}{2 a^{2}}\left(\frac{p}{\gamma-1}\right)_{i+1 / 2}+\rho_{i+1 / 2}-\frac{\gamma-1}{a^{2}}\left(\frac{p}{\gamma-1}\right)_{i+1 / 2}+\frac{\gamma-1}{2 a^{2}}\left(\frac{p}{\gamma-1}\right)_{i+1 / 2},
$$

where the values at $i+1 / 2$ are those from the characteristic variables in Equation (28) and the other values are from the transformation matrix. It is clear that if all three $p /(\gamma-1)$ terms are reconstructed consistently, the relevant terms cancel out, thus yielding a unique and consistent value for the density and constant velocity and pressure. It is interesting to note that if $\gamma$, in addition to $\rho$, is not constant, then the second $p /(\gamma-1)$ term in Equation (29) does not necessarily cancel the first and third terms, because its reconstructed value depends on the density weights.

Since the fluid is made of a single component, $R$ is constant. Thus, if the pressure remains constant, there will be no temperature errors.

\section{III.B. Advection of a material interface: form of the transport equation}

\section{III.B.1. Pressure viewpoint}

The pressure viewpoint is followed by Abgrall $(1996)^{1}$ to show that pressure oscillations may be generated at material interfaces separating fluids with different specific heats ratios. Substituting the relationship between pressure and internal energy (2) into the equation for the internal energy (22) yields

$$
\left(\frac{p}{\gamma-1}\right)_{i}^{n+1}=\left(\frac{p}{\gamma-1}\right)^{n}-\frac{\Delta t}{\Delta x} u D_{i}\left(\frac{p}{\gamma-1}\right)
$$

Thus, in order to maintain a constant pressure at time $n+1$, the following must hold:

$$
\left(\frac{1}{\gamma-1}\right)_{i}^{n+1}=\left(\frac{1}{\gamma-1}\right)_{i}^{n}-\frac{\Delta t}{\Delta x} u D_{i}\left(\frac{1}{\gamma-1}\right)
$$

In other words, $1 /(\gamma-1)$ must obey a transport equation in advection form in order to preserve the pressure equilibrium. It should be noted that this issue only occurs for problems with multiple fluids of different $\gamma$.

An underlying assumption for this result is that $D_{i}(p), D_{i}(u)=0$. As mentioned in the previous section, a reconstruction of the characteristic variables with Equation (30) alone does not prevent the generation of spurious pressure oscillations, because the $\gamma-1$ terms do not cancel in Equation (29).

Abgrall $(1996)^{1}$ first proposed to solve the transport equation in advection form for $1 /(\gamma-1)$ :

$$
\left(\frac{1}{\gamma-1}\right)_{i}^{n+1}=\left(\frac{1}{\gamma-1}\right)_{i}^{n}-\frac{\Delta t}{\Delta x} u D_{i}\left(\frac{1}{\gamma-1}\right)
$$




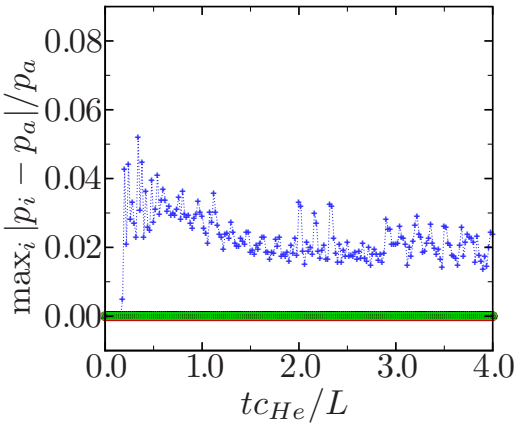

(a) Normalized $L_{\infty}$ error in pressure.

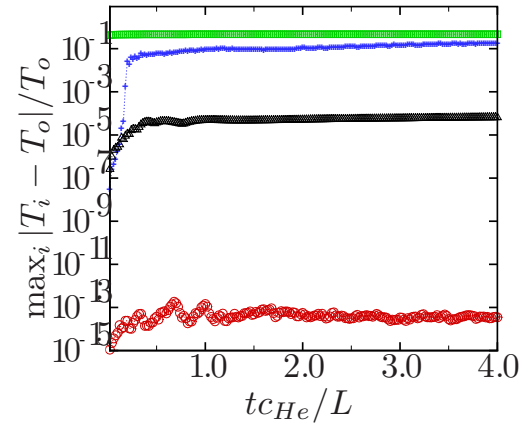

(b) Normalized $L_{\infty}$ error in temperature.

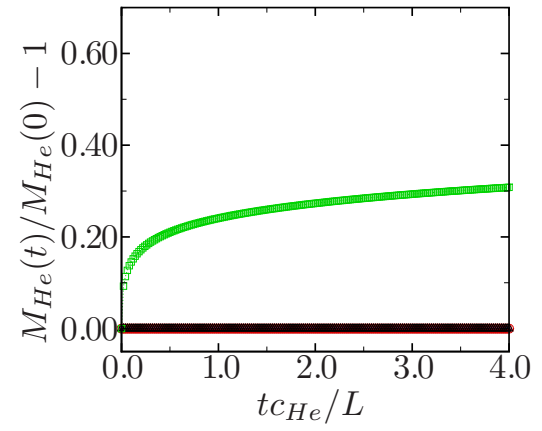

(c) Normalized error in mass of helium.

Figure 1. Time histories for the advection of a material interface between helium and nitrogen. Black solid line: exact solution; blue pluses: fully conservative scheme; grey open triangles: Abgrall's scheme; green open squares: Shyue's scheme; open circles: current scheme.

For the present purposes, there are several drawbacks to this scheme. Species conservation does not hold discretely since the equation is solved in advection form. Furthermore, in the case of different fluids with the same $\gamma$, an explicit relationship between the temperature and the mass fraction must be specified and an additional transport equation must be solved. Finally, small temperature oscillations are generated.

Shyue $^{11}$ proposes to solve an advection equation for $z$,

$$
z_{i}^{n+1}=z_{i}^{n}-\frac{\Delta t}{\Delta x} u D_{i}(z),
$$

instead of Equation (7), along with the following relationship between $1 /(\gamma-1)$ and $z$ :

$$
\frac{1}{\gamma-1}=\frac{z}{\gamma^{\alpha}-1}+\frac{1-z}{\gamma^{\beta}-1} .
$$

The discrete relationship between 1/( $\gamma-1)$ and $z$ in Equation (34) must be linear; otherwise, the relationship in Equation (31) cannot be recovered. There are several drawbacks to this scheme. Similarly to Abgrall's scheme, species conservation does not hold discretely. Furthermore, a modeling error, which does not vanish as the grid is refined, is introduced, as it is implicitly assumed that $M^{\alpha}=M^{\beta}$ in Equation (7). Finally, as shown below, a significant temperature spike is generated. The temperature is given by the ideal gas law (4), so that

$$
T_{i}^{n+1}=\frac{p}{\rho^{n+1}\left[z^{n+1} R^{\alpha}+\left(1-z^{n+1}\right) R^{\beta}\right]} \neq \frac{p}{(\rho R)^{n+1}}=T^{n} .
$$

Thus, a temperature error is generated. In practice, a spike forms at interfaces and is advected by the flow. However, because the temperature does not enter the problem, this issue is not relevant to the Euler equations; it only becomes problematic if the diffusion terms (particularly thermal conduction) in the NavierStokes equations are solved.

Abgrall and Shyue's schemes cannot be simply extended to high-order accurate reconstructions (e.g., WENO). A standard reconstruction of the conservative variables will invariably lead to spurious oscillations. In order to prevent these oscillations for high-order accurate schemes, a reconstruction of the average primitive variables must be carried out, as described in the previous section. The difference with the single-fluid case resides in the fact that the second term in brackets in equation (26) is no longer zero. The relationship in equation (31) is recovered by advecting $1 /(\gamma-1)$.

Results from the advection of a material interface are considered in Figure 1, which shows time histories for the advection of a material interface. Three different schemes are shown: fully conservative scheme, Abgrall's scheme and Shyue's scheme. The errors in the pressure when using the standard fully conservative scheme are approximately 5\%. Temperature errors are observed in all the schemes except for the present scheme; the errors generated by Abgrall's scheme are quite small. When using Shyue's scheme, these errors may be up to $50 \%$. The error in the mass of helium increases significantly with time when using Shyue's scheme. Abgrall's scheme does not generate significant errors in the mass of helium. 


\section{III.B.2. Temperature viewpoint}

Alternately, the temperature viewpoint can be followed to prevent pressure oscillations at material interfaces. ${ }^{8}$ Substituting the relationship between temperature and internal energy (4) into the equation for the internal energy (22) yields

$$
\left(\rho c_{v} T\right)^{n+1}=\left(\rho c_{v} T\right)^{n}-\frac{\Delta t}{\Delta x} u D_{i}\left(\rho c_{v} T\right) .
$$

Thus, in order to maintain a constant temperature, the following must hold:

$$
\left(\rho c_{v}\right)_{i}^{n+1}=\left(\rho c_{v}\right)_{i}^{n}-\frac{\Delta t}{\Delta x} u D_{i}\left(\rho c_{v}\right) .
$$

This equation can be re-arranged into a transport equation in conservative form:

$$
\left[\left(c_{v}^{\alpha}-c_{v}^{\beta}\right)(\rho z)^{n+1}+c_{v}^{\beta} \rho^{n+1}\right]=\left[\left(c_{v}^{\alpha}-c_{v}^{\beta}\right)(\rho z)^{n}+c_{v}^{\beta} \rho^{n}\right]-\frac{\Delta t}{\Delta x} u\left[\left(c_{v}^{\alpha}-c_{v}^{\beta}\right) D_{i}(\rho z)+c_{v}^{\beta} D_{i}(\rho)\right],
$$

provided $D_{i}$ acting on $\rho z$ is the same as $D_{i}$ acting on $\rho$. If this is the case, pressure equilibrium is maintained:

$$
p^{n+1}=\left[\left(R^{\alpha}-R^{\beta}\right) \rho z_{i}^{n}+R^{\beta} \rho_{i}^{n}\right] T-\frac{\Delta t}{\Delta x} u D_{i}\left[\left(R^{\alpha}-R^{\beta}\right) \rho z+R^{\beta} \rho\right] T=p,
$$

since the pressure is uniform initially. Maintaining the pressure equilibrium requires that $\rho R T$ remain constant, but not that $\rho R$ and $T$ are both constant. In order to additionally maintain the temperature equilibrium, $\rho R$ must both be constant at every stage of the process. This can be achieved by ensuring that $D_{i}$ acting on $\rho z$ is the same as $D_{i}$ acting on $\rho$. This condition is not trivial for general reconstructions; for high-order accurate WENO, a sufficient condition to meet this criterion is that the nonlinear weights for $\rho$ and $\rho z$ be the same, e.g., using the weights of $\rho$ for $\rho z$ or some linear combination of the two.

An advantage of this procedure is that the transport equation is solved in conservative form, so that the mass of each species is conserved. However, this scheme generates spurious pressure oscillations when applied to cases in which the temperature is discontinuous as well, i.e., contact discontinuities separating different fluids. A means to prevent this drawback is proposed in the next section.

\section{III.C. Advection of a contact discontinuity separating different fluids: the proposed scheme}

The advection of a contact discontinuity separating different fluids was analyzed by Johnsen et al. ${ }^{8}$ In this case, $u$ and $p$ are constant, but $z$ and $T$, and therefore $\rho$, are not. The advantages of the pressure and temperature viewpoints for a material interface can be combined to simulate contact discontinuities separating different fluids without generating pressure and temperature errors: the Euler equations are solved along with two transport equations, one in conservative form for $\rho z$, which is used to compute the temperature and mass fraction in the diffusive terms only, and one in advection form for $1 /(\gamma-1)$, which is used to compute the pressure in the convective terms only. Though the temperature and mass fraction do not enter the Euler equations as such, their accurate calculation is necessary for the relevant terms in derivatives of these values in diffusive terms. The equation for $1 /(\gamma-1)$ can be viewed as an equation for a pressure correction that maintains the pressure equilibrium when needed; even though a discontinuity in $1 /(\gamma-1)$ may represent an interface, interfaces are defined by discontinuities in $z$. This framework will conserve the mass of each species, preserve the pressure equilibrium (and temperature equilibrium in the case of a material interface), and does not introduce a modeling error. The proposed scheme can be thought of as an extension to Abgrall's scheme in which an additional transport equation for the mass fraction is solved in conservative form and consistently with the continuity equation.

One caveat with the proposed formulation is that Equation (7) is not explicitly enforced; if it were, the problem would become over-specified. Hence, a potential difficulty is that interfaces defined by $z$ may not be consistent with those defined by $1 /(\gamma-1)$ in more complicated flows, e.g., turbulence. It can be shown that from the present (conserved) scheme

$$
z_{i}^{n+1}=z_{i}^{n}+\frac{\left[(\rho z)_{i}^{n+1}-(\rho z)_{i}^{n}\right]-z_{i}^{n}\left(\rho^{n+1}-\rho^{n}\right)}{\rho_{i}^{n+1}}=z_{i}^{n}-\frac{\Delta t}{\Delta x} u \frac{z_{i} D_{i}(\rho)-D_{i}(\rho z)}{\rho_{i}^{n+1}} \approx z_{i}^{n}-\frac{\Delta t}{\Delta x} u D_{i}(z) .
$$

Thus, $z_{i}^{n+1}$ obtained from the advection form of the transport equation for $1 /(\gamma-1)$ tends to the value obtained from the conservative form as the grid and time step are refined. In the laminar problems described 


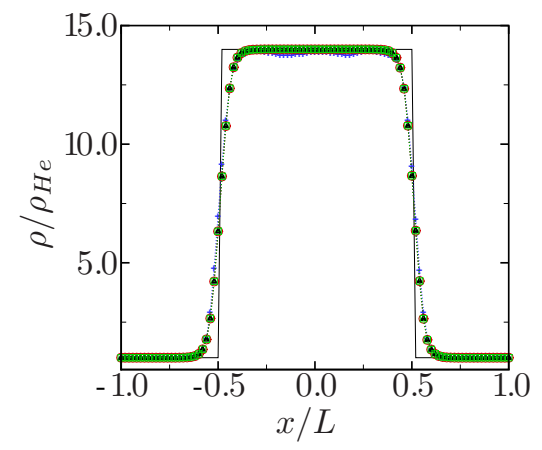

(a) Density.

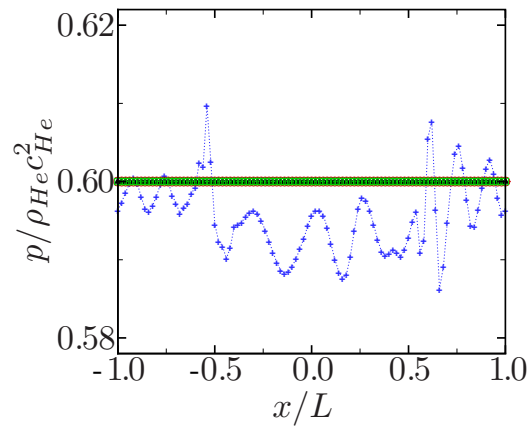

(b) Pressure.

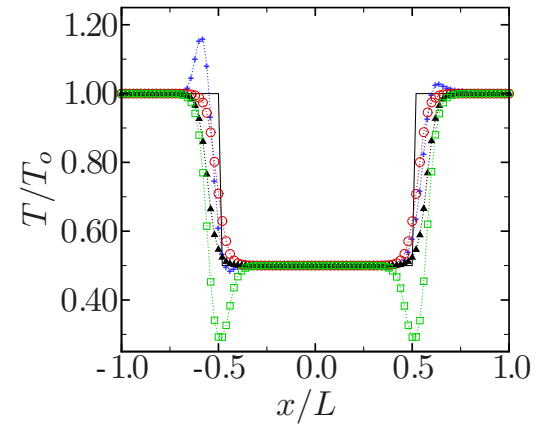

(c) Temperature.

Figure 2. Profiles at $t c_{H e} / L=4$ for the advection of a material interface between helium and nitrogen. Black solid line: exact solution; blue pluses: fully conservative scheme; grey open triangles: Abgrall's scheme; green open squares: Shyue's scheme; open circles: current scheme.

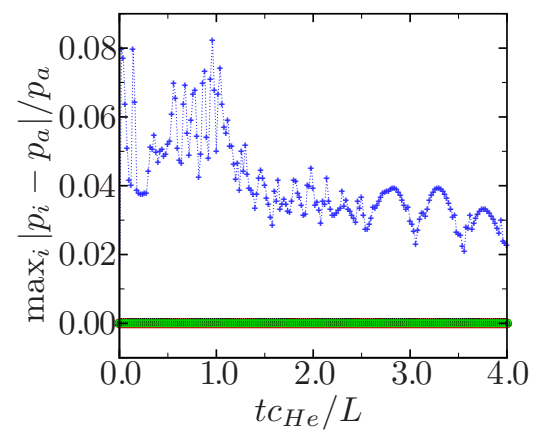

(a) Normalized $L_{\infty}$ error in pressure.

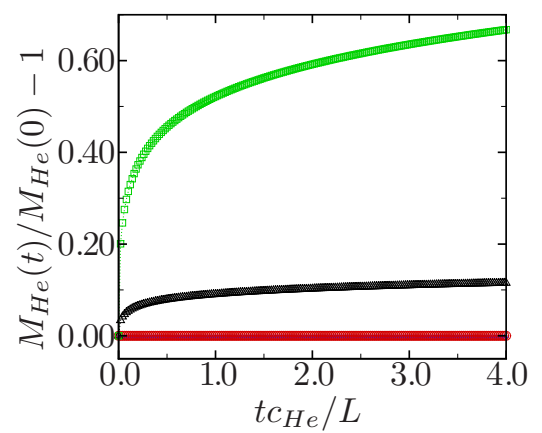

(b) Normalized error in mass of helium.

Figure 3. Time histories for the advection of a material interface between helium and nitrogen. Black solid line: exact solution; blue pluses: fully conservative scheme; grey open triangles: Abgrall's scheme; green open squares: Shyue's scheme; open circles: current scheme.

in this article, the present method shows good results. However, additional investigations are required to determine whether the present scheme is applicable to fully turbulent flows.

Figure 2 shows the density, pressure and temperature profiles at the final time for the advection of a contact discontinuity between two different gases. The errors in the pressure when using the standard fully conservative scheme grow up to approximately $8 \%$. The proposed scheme is the only one that prevents pressure, temperature and species conservation errors.

\section{Conclusion}

Numerical errors generated by high-order accurate shock-capturing schemes for problems with density discontinuities, but with constant velocity and pressure, were studied theoretically. A unifying theory describing the pressure and temperature errors was provided. Generally, weighted essentially non-oscillatory schemes in which the primitive variables are reconstructed prevent spurious pressure oscillations. Temperature errors are further avoided by reconstructing the density and mass fraction in a consistent fashion.

\section{Acknowledgements}

The authors are grateful for useful discussions with Dr. V. Terrapon, Dr. C. Liang, Dr. S. Kawai, and Professor S. K. Lele. This work was supported by DOE-SciDAC2 (Grant DE-FC02-06-ER25787). 


\section{References}

${ }^{1} \mathrm{R}$. Abgrall, How to prevent pressure oscillations in multicomponent flow calculations: a quasi conservative approach, $J$. Comput. Phys. 125, 150 (1996).

${ }^{2}$ M. Brouillette, The Richtmyer-Meshkov instability, Annu. Rev. Fluid Mech. 34 (2002) 445-468.

${ }^{3}$ B. Einfeldt, C. D. Munz, P. L. Roe, and B. Sjögreen, On Godunov-type methods near low densities, J. Comput. Phys. 92, 273 (1991).

${ }^{4}$ S. Gottlieb and C. W. Shu, Total variation diminishing Runge-Kutta schemes, Math. Comp. 67, 73 (1998).

${ }^{5}$ A. Harten, P. D. Lax, and B. van Leer, On upstream differencing and Godunov-type schemes for hyperbolic conservation laws, SIAM Review 25, 35 (1983).

${ }^{6}$ E. Johnsen and T Colonius, Implementation of WENO schemes in compressible multicomponent flows J. Comp. Phys. 219, 715-732 (2006).

${ }^{7}$ E. Johnsen, On the treatment of contact discontinuities using WENO schemes, submitted (2011).

${ }^{8}$ E. Johnsen, J. Larsson and F. Ham, Preventing numerical errors generated by WENO-based interface-capturing schemes in compressible multi-material flows, submitted (2011).

${ }^{9}$ X. D. Liu, S. Osher, and T. Chan, Weighted essentially non-oscillatory schemes, J. Comput. Phys. 115, 200 (1994).

${ }^{10} \mathrm{R}$. Saurel and R. Abgrall, A simple method for compressible multifluid flows, SIAM J. Sci. Comput. 21, 1115 (1999).

${ }^{11}$ K. M. Shyue, An efficient shock-capturing algorithm for compressible multicomponent problems, J. Comput. Phys. 142, 208 (1998) 